\title{
SARS-CoV-2 serology: Test, test, test, but interpret with caution!
}

\author{
Authors: William H Bermingham, ${ }^{A}$ Thomas Wilding, ${ }^{B}$ Sarah Beck ${ }^{C}$ and Aarnoud Huissoon ${ }^{D}$
}

\begin{abstract}
SARS-CoV-2 serological tests are a subject of intense interest and have the potential to significantly enhance the diagnostic capability of healthcare services in the current pandemic. However, as with all novel assays, significant validation is required to understand the clinical relevance of results. We present the first study to assess clinician interpretation of SARS-CoV-2 serology scenarios. We identify common key assumptions regarding patient infectivity and protection that are not currently supported by the SARS-CoV- 2 evidence base. In this rapidly developing field, we therefore strongly recommend serological assay results are accompanied by clear interpretive support from laboratory and infectious diseases specialists.
\end{abstract}

KEYWORDS: COVID-19, SARS-CoV-2, serology, interpretation of laboratory results

DOI: $10.7861 /$ clinmed.2020-0170

\section{Serological testing in SARS-CoV-2}

The novel coronavirus SARS-CoV-2, manifesting clinically as the disease named COVID-19, has caused a global pandemic. As of 4 May 2020 there have been 3,442,234 confirmed cases and 239,740 fatalities reported across 215 countries.

Diagnostic assays for SARS-CoV-2 have been in rapid development, and large studies examining sensitivity and specificity for all platforms are understandably lacking at this early stage. Currently, nucleic acid amplification tests (NAATs) provide a direct method to detect the presence of the SARS-CoV-2 RNA genome. These tests are in widespread diagnostic use to identify active infection. However, in isolation, these assays are not comprehensive. There is an urgent need to expand diagnostic capability to include indirect detection methods, which may be

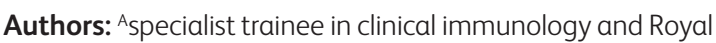
College of Physicians chief registrar, University Hospitals Birmingham NHS Foundation Trust, Birmingham, UK; ${ }^{B}$ senior clinical scientist in immunology, University Hospitals Birmingham NHS Foundation Trust, Birmingham, UK; ' Consultant clinical scientist in immunology, University Hospitals Birmingham NHS Foundation Trust, Birmingham, UK; ' ${ }^{D}$ consultant immunologist, University Hospitals Birmingham NHS Foundation Trust, Birmingham, UK applicable both during active infection and for the identification of previously infected individuals who were not tested at the time of their acute illness. This has led to intense interest in the potential of serological assays (Fig 1). ${ }^{2-4}$

Serological testing identifies host humoral immune responses to an infection. In principle, this has the potential for broad clinical applications, including studying the immune response, epidemiological applications (such as establishing rates of infection and fatality, identifying asymptomatic cases, and carrying out contact tracing, transmission pattern analysis and patient contact studies), and identifying those in the population who may be immune. ${ }^{5}$ However, this relies on an understanding of the basic immunobiology of an infection, coupled with robust assay validation. It is perhaps unsurprising that even well-established assays, for example those for acute Epstein-Barr virus, hepatitis and cytomegalovirus infections, can present challenges in the interpretation of results for clinical application. The ability to correctly identify all cases of infection (sensitivity) and to discriminate between cross-reactive viruses and other antigens (specificity) vary widely between tests, meaning that it is not possible to make global assumptions regarding the interpretation of vaccine serology. ${ }^{6,7}$ Longrecognised conditions further benefit from established diagnostic algorithms, such as those detailed in the Public Health England Standards for Microbiology Investigations (UK SMI). However, such interpretive support does not yet exist for SARS-CoV-2. ${ }^{8}$

Early studies of SARS-CoV-2 immunobiology have identified the emergence of specific immunoglobulin M (IgM)/IgA and IgG SARSCoV-2 antibodies at approximately day 5 and day 14 of infection respectively. ${ }^{9}$ However, recent data have challenged the assumed principles of sequential virus-specific antibody seroconversion from an early IgM response followed by a later emergence of IgG. Long et al instead describe three distinct patient groups: ${ }^{10}$

> synchronous seroconversion of IgG and IgM

$>$ IgM seroconversion earlier than that of IgG

$>$ IgM seroconversion later than that of IgG.

Furthermore, data are not yet available for specific populations who may not mount a specific antibody response, such as those with immunodeficiencies.

The development of serological assays has mainly focused on antibodies directed against the SARS-CoV-2 spike and nucleocapsid proteins. Such antibodies have been shown to neutralise virus in vitro.11,12 However, a significant number of patients experiencing COVID-19 may generate low titres of specific antibodies, presenting a challenge to detection. ${ }^{13}$ Differing 

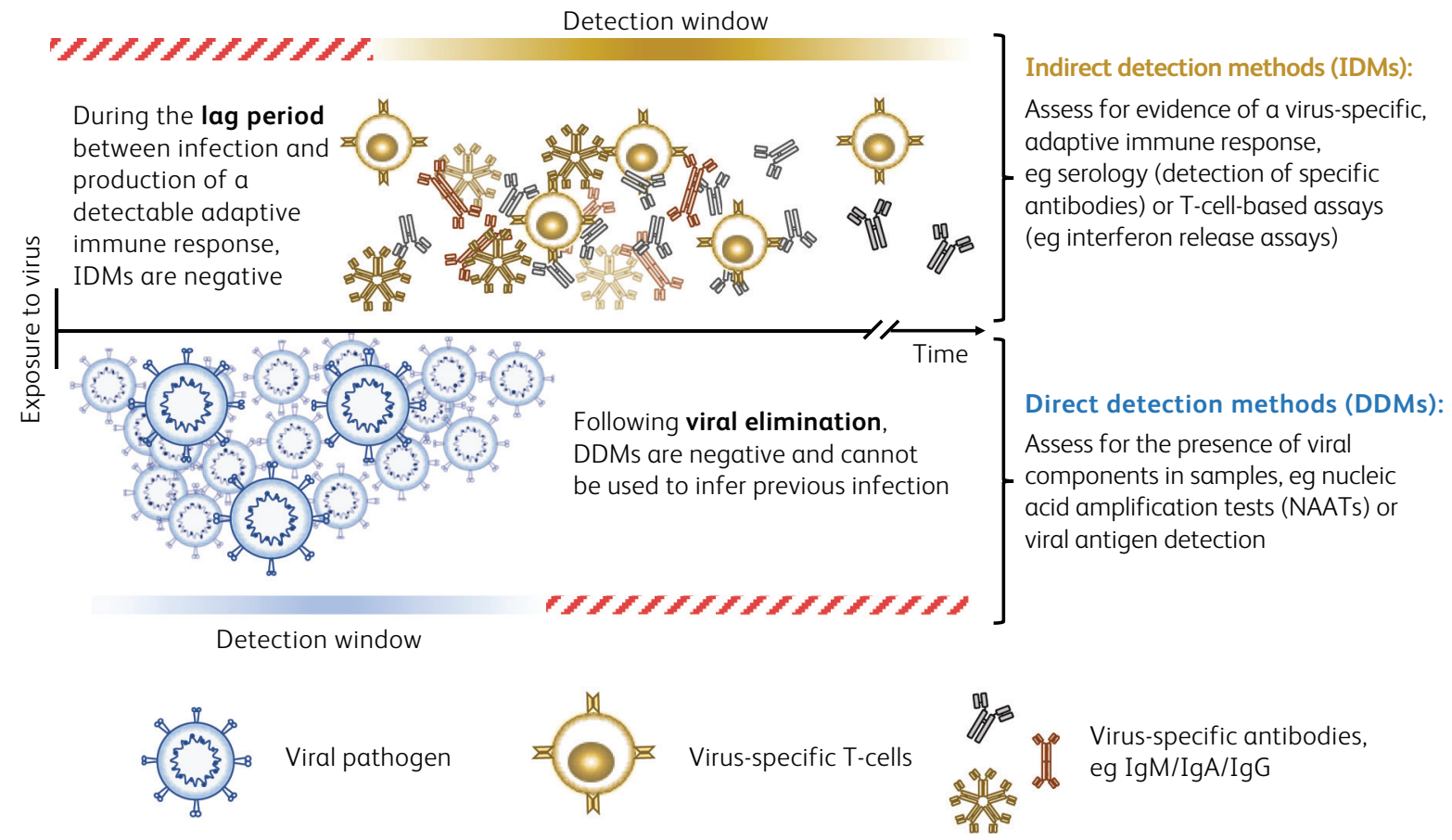

Virus-specific antibodies, eg IgM/IgA/IgG

Fig 1. Summary of direct and indirect detection methods.

patterns of antibody detection have also been associated with both viral clearance and clinical outcomes. ${ }^{14,15}$ Overall, at this early stage in the pandemic, the evidence base relating to SARS-CoV-2 remains limited. It is noteworthy that many studies await peer review, with 2,721 preprint articles available on medrxiv and biorxiv websites (www.medrxiv.org, www.biorxiv.org).

\section{Clinician interpretation of IgM and IgG serological results in SARS-CoV-2}

With large-scale implementation of novel serology assays likely to be imminent, how the results are used will have implications for both individual patient care and public health measures. To better understand how SARS-CoV-2 IgM and IgG results may be interpreted by clinicians, a survey was designed using the SurveyMonkey web-based platform (SurveyMonkey, San Mateo, USA; www.surveymonkey.com) (supplementary material S1). An online survey link was distributed to clinicians and clinical scientists in the UK via existing professional networks, constituting a 'snowball' sampling method. The survey was designed to be appropriate to the responding clinicians by presenting serological results as these may be encountered in routine clinical practise. Due to technological limitations of the survey platform used, this initial survey was closed once a maximum of 100 responses had been received. Grades and specialities of responders are summarised in Table 1. Results were collected between 25 March 2020 and 31 March 2020. During this period, serology testing for SARS-CoV-2 was not generally available in the UK.

Responders were asked to interpret four result combinations for SARS-CoV-2-specific IgM and IgG serology, first in isolation and then with the addition of a clinical scenario stating 'active symptoms consistent with COVID-19'. Responders could select all statements that they felt were appropriate to each scenario. Data were analysed using Graphpad Prism 8 (GraphPad Software, San Diego, California USA, www.graphpad.com) and are summarised in Fig 2. An optional free-text comment box was provided for each scenario and responses recorded (supplementary material S2).

Interpreting serology results alone and in the context of relevant symptoms resulted in notable variation. This was particularly marked for IgM- IgG- and IgM- IgG + scenarios. $17 \%$ of responders classed a patient with negative serology (IgM- IgG-) as having 'No COVID-19' despite the presence of active symptoms. Also, $40 \%$ considered patients to have 'cleared COVID-19' despite active symptoms in the context of serology demonstrating IgM- IgG+.

Links between serology and a patient's risk of infection or their ability to infect others have not been clearly established for SARSCoV-2. Yet, across all serology and serology plus clinical scenarios, a mean of $57 \%$ (SD 17\%) of participants selected statements inferring a patient's infectivity status, and 41\% (SD 18\%) selected statements inferring immunity status. In clinical practice, misplaced confidence in the interpretation of serology could lead to errors of management. 22/91 of the free-text comments queried assay performance, for example wanting to review sensitivity/specificity data.

\section{Conclusions}

The rapid development and implementation of a range of diagnostic assays is undoubtedly an essential part of the coordinated response to a new pathogen. However, the limitations of novel assays and of clinicians' understanding of these must be considered. ${ }^{4,5}$ To our knowledge, this is the first study to investigate clinicians' interpretive response to novel SARS-CoV-2 serology. There are significant limitations to our study design, both in our 
Table 1. Summary of survey responder demographics

Specialities of clinicians who undertook the survey*

Acute medicine

Anaesthetics

Paediatric psychiatry

Clinical immunology

Core medical training

Citical care

GP

Dermatology

Emergency medicine

Endocrinology

ENT

Foundation programme

Gastroenterology

General surgery

Geriatrics

Gynaecology

Haematology

Histopathology

Infectious diseases

International training fellow

Medical microbiology

General internal medicine 3

Nephrology

Neurosurgery

Specialities of clinicians who undertook

the survey*

Oncology

Paediatrics

Palliative medicine

Psychiatry

Radiology

Respiratory medicine

Rheumatology

HSST programme 3

General surgery

Trust grade doctor 1

Clinical scientist

Reported training grade of clinicians who undertook the survey ${ }^{\dagger}$

Advanced nurse practitioner

Clinical scientist

Core trainee/senior house officer 21

Consultant

Foundation trainee

GP

Specialist trainee ST3+ (registrar)

Staff grade

Trainee clinical scientist

Number of responders

4

8

1

11

4

3

11

1

1

6

1

4

4

2

Number of responders

1

1

1

1

2

2

3

1

2

Number of responders

1

6

21

6

7

5

50

1

3

${ }^{*}$ Clinicians from a total of 35 different specialities provided responses. ${ }^{+} 50 \%$ of responders were of UK specialist trainee grades $\geq$ ST3.

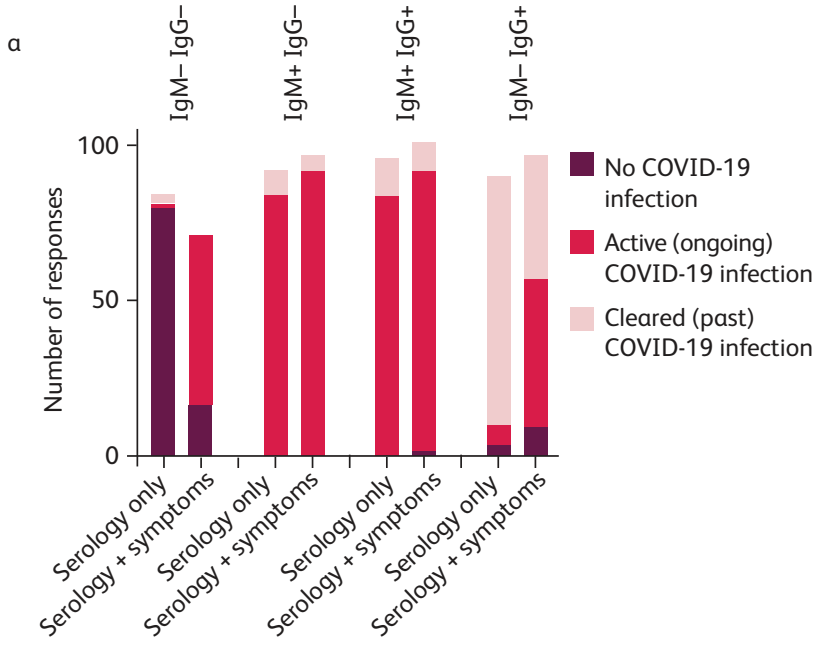

b
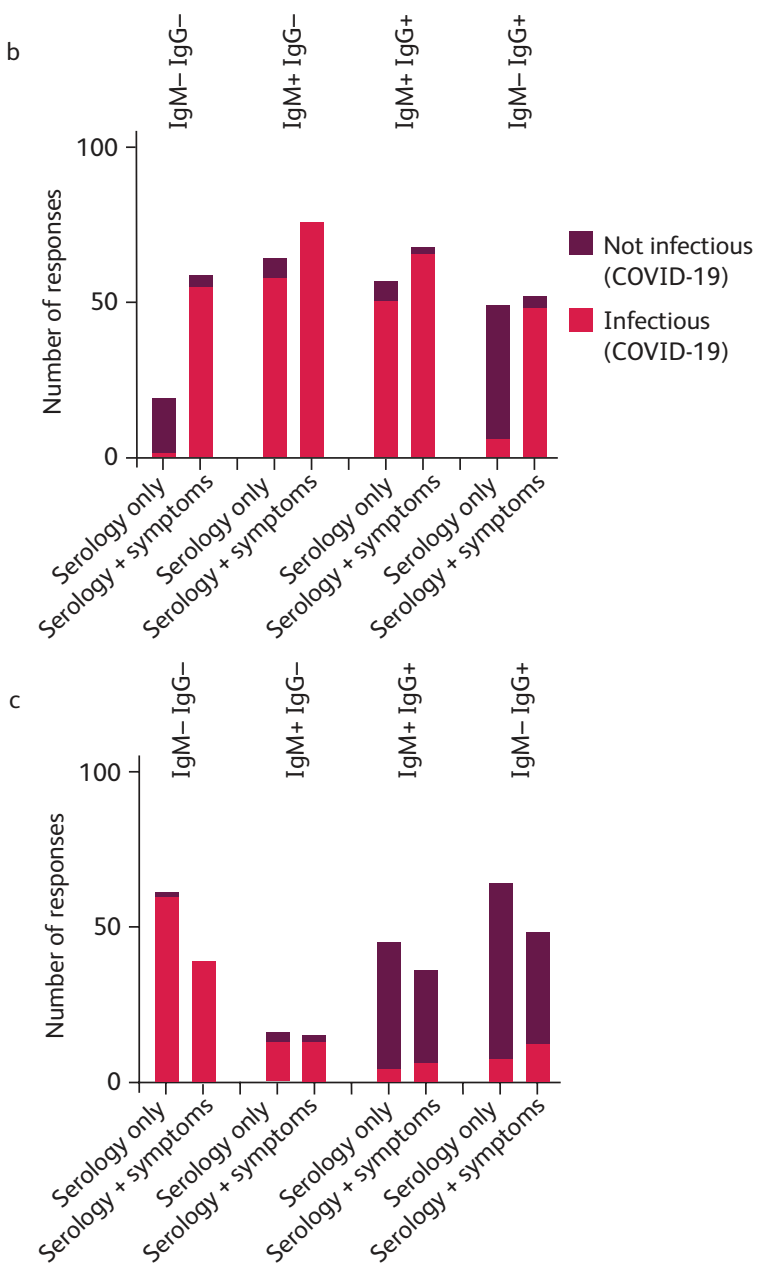

Immune (not at risk of future infection with COVID-19)

Not immune (at risk of future infection with COVID-19)

Fig 2. Summary of survey responses. For each scenario, responders were asked to select all statements they felt were appropriate to the serology result with and without associated clinical details of 'active symptoms consistent with COVID-19'. a) Responses inferring the patient's SARS-CoV-2 infection status. b) Responses inferring the patient's risk of infecting others with SARS-CoV-2. c) Responses inferring the patient's risk of future infection with SARS-CoV-2. 


\section{Box 1. Examples of interpretative comments that} may be useful in reporting SARS-CoV-2 serology

These must be modified to reflect the validation characteristics and specifications of the assay system used.

$>$ SARS-CoV-2 IgG antibodies can be found in A-B\% of persons $>2$ weeks after infection.

$>$ An increase in SARS-CoV-2 antibodies supports a diagnosis of recent infection.

$>$ SARS-CoV-2 antibody results cannot be used to infer an individual's infectivity.

> SARS-CoV-2 antibody results are not yet known to correlate with immunity.

modest number of survey responses and the necessity for rapid design and implementation due to the evolving nature of the pandemic. As free text comments were optional, analysis of these is also limited. However, we highlight that there is likely to be marked variation in the clinical interpretation of SARS-CoV-2 serology results as they become available. Further research in this area is urgently warranted, as this may have serious implications for ongoing public health efforts to maintain social distancing measures and the isolation of patients affected by COVID-19. Proactive interpretive support, which includes 'narrative comments' from laboratory and infectious diseases specialists, is strongly recommended (Box 1).

\section{Supplementary material}

Additional supplementary material may be found in the online version of this article at www.rcpjournals.org/clinmedicine:

S1 - Survey structure

S2 - Free text comments submitted by survey responders

\section{References}

1 World Health Organization. Coronavirus disease (COVID-19) pandemic. WHO, 2020. Available from www.who.int/emergencies/ diseases/novel-coronavirus-2019 [Accessed 4 May 2020].

2 World Health Organization. Laboratory testing for coronavirus disease 2019 (COVID-19) in suspected human cases. WHO, 2020. www.who.int/publications-detail/laboratory-testing-for-2019novel-coronavirus-in-suspected-human-cases-20200117 [Accessed 11 April 2020].

3 Patel R, Babady E, Theel ES et al. Report from the American Society for Microbiology COVID-19 International Summit, 23 March
2020: Value of diagnostic testing for SARS-CoV-2/COVID-19. mBio 2020;11:e00722-20.

4 Egner W, Beck S, Chopra C et al. Statement from RCPath's Immunology Specialty Advisory Committee on COVID-19/ SARS CoV2 antibody evaluation. Royal College of Pathologists, 2020. www.rcpath.org/uploads/assets/194ed03e-9b0a-4f658208563290fb848e/3aeb35a1-97d1-4143-92440dc0aa42c5e8/ G211-RCPath-Immunology-SAC-statement-on-COVID-19-SARSCoV2-antibody-evaluation.pdf [Accessed 11 April 2020].

5 Meyer B, Drosten C, Müller MA. Serological assays for emerging coronaviruses: Challenges and pitfalls. Virus Res 2014;194:175-83.

6 Klutts JS, Ford BA, Perez NR, Gronowski AM. Evidence-based approach for interpretation of Epstein-Barr virus serological patterns. J Clin Microbiol 2009;47:3204-10.

7 Landry ML. Immunoglobulin M for acute infection: True or false? Clin Vaccine Immunol 2016;23:540-5.

8 Public Health England. Standards for microbiology investigations. PHE, 2014. www.gov.uk/government/collections/standards-for-microbiology-investigations-smi\#uk-smi-supporting-information [Accessed 4 May 2020].

9 Guo L, Ren L, Yang S et al. Profiling early humoral response to diagnose novel coronavirus disease (COVID-19). Clin Infect Dis 2020; ciaa310 [Epub ahead of print].

10 Long Q-X, Liu B-Z, Deng H-J et al. Antibody responses to SARS-CoV-2 in patients with COVID-19. Nat Med 2020;26:845-8.

11 Haveri A, Smura T, Kuivanen $S$ et al. Serological and molecular findings during SARS-CoV-2 infection: the first case study in Finland, January to February 2020. Eur Commun Dis Bull 2020;25:2000266.

12 Okba Ni MA, Muller MA, Li W et al. SARS-CoV-2 specific antibody responses in COVID-19 patients. medRxiv 2020;2020.03.18. 20038059

13 Wu F, Wang A, Liu M et al. Neutralizing antibody responses to SARS-CoV-2 in a COVID-19 recovered patient cohort and their implications. medRxiv 2020; 2020.03.30.20047365.

14 Zhao J, Yuan Q, Wang H et al. Antibody responses to SARS-CoV-2 in patients of novel coronavirus disease 2019. medRxiv 2020:2020.03.02.20030189.

15 Kai-wang K, Tak O, Tsang Y et al. Temporal profiles of viral load in posterior oropharyngeal saliva samples and serum antibody responses during infection by SARS-CoV-2: an observational cohort study. Lancet Infect Dis 2020;20:565-74.

Address for correspondence: Dr William Hywel Bermingham, Department of Clinical Immunology and Allergy, Heartlands Hospital, Bordesley Green East, Birmingham B9 5SS, UK. Email: william.bermingham@nhs.net 